\title{
HISTOIRE DE LA LOGIQUE FLOUE UNE APPROCHE SOCIOLOGIQUE DES PRATIQUES DE DÉMONSTRATION
}

\author{
Claude RosenTAL
}

RÉsumÉ : Cet article aborde la question générale du développement d'une histoire sociale des mathématiques et de la logique, à partir d'un cas historique. Il vise à rendre compte de certains traits de l'histoire récente de la logique floue. À cette fin, il met en œuvre une sociologie des pratiques de démonstration et une approche fondée sur l'analyse matérielle du travail logique, notamment de l'activité d'écriture et de lecture. Il esquisse la construction d'une histoire sociale des formes de démonstration.

Mots-CLÉs : logique floue, histoire de la logique, usages sociaux des démonstrations, sociologie des formalismes, démos.

ABSTRACT : Starting from an historical case study, this article addresses the general issue of the development of a social history of mathematics and logic. It aims at giving an account of certain features of recent history of fuzzy logic. For this purpose, it develops an approach based on a sociology of methods of demonstration and on the material analysis of logical activity, in particular of writing and reading activities. The formation of a social history of forms of demonstration is outlined.

KErwords : fuzzy logic, history of logic, social use of demonstrations, sociology of formalisms, demos.

Revue de synthèse : $4^{\text {e S. }} \mathrm{n}^{\circ}$ 4, oct.-déc. 1998, p. 575-602. 
Zusammenfassung: Ausgehend von einer historischen Fallstudie wird in diesem Artikel das Problem der Entwicklung einer Sozialgeschichte der Mathematik untersucht, wobei einige Aspekte der neueren Geschichte der unbestimmten Logik berücksichtigt werden. Das geschieht anhand einer Soziologie der Beweisführung und mit einem Ansatz, der auf der materiellen Analyse des logischen Vorgehens, insbesondere des Schreibens und des Lesens, beruht. Es wird skizziert, wie der Aufbau einer Sozialgeschichte der Beweisformen aussehen könnte.

STICHWÖRTER : unbestimmte Logik, Geschichte der Logik, sozialer Gebrauch von Beweisen, Soziologie der Formalismen, demos.

Claude Rosental, sociologue, est maître de conférences à l'université de Rouen. Il étudie les usages sociaux des démonstrations, en logique et dans le cadre d'autres activités humaines. Il est l'auteur d'une thèse intitulée : "L'émergence d'un théorème logique. Une approche sociologique des pratiques contemporaines de démonstration » (1996).

Aaresse : Département de sociologie, UFR de sociologie, psychologie et sciences de l'éducation, Université de Rouen, rue Lavoisier, 76821 Mont-Saint-Aignan Cedex.

Courrier électronique : rosental@epeire.univ-rouen.fr 
"The boat of uncertainty reasoning is being rebuilt at sea. Plank by plank fuzzy theory is beginning to gradually shape its design. Today only a few fuzzy planks have been laid. But a hundred years from now, a thousand years from now, the boat of uncertainty reasoning may little resemble the boat of today. Notions and measures of overlap $A \cap A^{c}$ and underlap $A \cup A^{c}$ will have smoothed its rudder. Amassed fuzzy applications, hardware, and products will have broadened its sails. And no one on the boat will believe that there was a time when a concept as simple, as intuitive, as expressive as a fuzzy set met with such impassioned denial ${ }^{1}$. "

Comment mettre en œuvre une histoire sociale des mathématiques et de la logique ? Face à cette question, cet article vise à discuter et à explorer certaines options méthodologiques, en les déployant sur un cas historique. Il s'agit ainsi de rendre compte du développement d'une logique particulière, répondant au nom de «logique floue ». Ce travail s'appuiera sur l'analyse de documents d'archives, mais aussi sur des entretiens et des observations empiriques réalisés dans la première moitié des années 1990 en France et aux États-Unis.

L'historiographie sur la logique floue est aujourd'hui circonscrite à une série de repères qui appartiennent avant tout à l'histoire des idées propres à ce domaine. Quelques récits mettent en scène d'autres éléments, relevant des conditions techniques, industrielles et institutionnelles du développement de la logique floue. Toutefois, puisque des narrations qui intègrent et surtout articulent l'ensemble de ces données n'ont pu être identifiées, ce sont les possibilités d'un traitement global et homogène de ces dernières qu'il conviendra d'explorer. À cette fin, sera mise en œuvre une approche fondée sur l'analyse matérielle du travail logique, notamment des pratiques d'écriture et de lecture, et une sociologie des pratiques de démonstration.

MODES DE PRÉSENTATION DE L'HISTOIRE DE LA LOGIQUE FLOUE

Quel est donc tout d'abord l'état actuel de l'historiographie sur la logique floue? Quelles descriptions y sont faites des principes de cette théorie logique? Il convient en premier lieu de préciser que les tentatives

1. Pour plus de précisions sur les références citées en notes, se reporter à la bibliographie finale, p. 600-602. Ici, Kosko, 1990, p. 239. 
d'histoire de la logique floue que nous avons pu identifier ont été rédigées non pas par des historiens des sciences, mais par des contributeurs immédiats aux travaux et débats qui caractérisent ce domaine de recherche depuis la fin des années 1960. Si l'essor de la logique floue ne semble pas avoir jusqu'ici suscité d'intérêt auprès des historiens, les narrations à ce sujet, plus souvent orales qu'écrites, constituent depuis le début des années 1990 une activité notable pour les partisans de la logique floue, dotée d'enjeux spécifiques ${ }^{2}$. Ces derniers proposent des histoires et par là même des définitions de leur objet à la fois diverses, évolutives et concurrentes.

Par-delà la diversité des histoires de la logique floue que l'on peut ainsi rencontrer, la plupart d'entre elles se présentent à la manière d'une histoire des idées. Il s'agit généralement d'histoires de l'invention d'une théorie ${ }^{3}$. Ces récits insistent le plus souvent sur le fait que la logique floue permet de prendre en compte une infinité de degrés de vérité contrairement à la logique binaire, qui ne permet d'exprimer que deux valeurs de vérité (« vrai » et « faux»).

De telles narrations associent communément l'invention de la logique floue à un premier article publié en 1965 par un professeur d'automatique de l'université de Californie à Berkeley, Lotfi A. Zadeh ${ }^{4}$. Plusieurs de ces récits sont structurés par le déploiement d'un registre spécifique, celui des motivations du développement d'une théorie logique, propres à son inventeur. Dans ce cas de figure, la logique floue est fréquemment associée au souci de Zadeh de construire un formalisme permettant d'effectuer des raisonnements qualifiés de qualitatifs. La capacité à effectuer de tels raisonnements étant alors présentée comme " naturelle » chez les êtres humains, ces narrations insistent au bout du compte sur la volonté originelle de Zadeh de doter les ordinateurs de facultés proprement humaines. Le passage suivant constitue une bonne illustration de ce type de présentation ${ }^{5}$ :

"The theory of Fuzzy Logic was introduced to the world by professor Lotfi A. Zadeh of the University of California at Berkeley. Professor Zadeh observed that conventional computer logic is incapable of manipulating data representing subjective or vague human ideas, such as "an attractive person" or "pretty hot". Computer logic previously envisioned reality only in such simple terms, as on or off, yes or no, and black or white. Fuzzy Logic was designed to allow computers to determine valid distinctions among data with shades of gray, working similarly in essence to the processes which occur in human rea-

2. Sur une situation comparable, voir GoldsTEIN, 1995.

3. Pour une analyse systématique des modes de construction de l'histoire d'une invention, par-delà les cas ici abordés, voir Mialet, 1994.

4. ZADEH, 1965.

5. Voir Archives du forum électronique d'Internet « comp.ai.fuzzy », article $\mathbf{n}^{\circ} 124$. 
soning. Accordingly, Fuzzy technologies are designed to incorporate Fuzzy theories into modern control and data processing, to create more user-friendly systems and products. "

D'autres chercheurs en logique floue, adoptant une posture plus épistémologique, inscrivent le développement de leur objet dans le cadre de l'émergence récente et plus générale d'un nouveau champ de recherches, portant sur le traitement de diverses formes de connaissances imparfaites. Selon eux, la logique floue ferait partie d'un ensemble d'approches concurrentes ou complémentaires à la théorie des probabilités, qui était jusqu'à peu en situation de monopole sur ce champ. Ces chercheurs placent généralement dans cet ensemble diverses approches de l'incertain, du vague, de l'imprécision, de l'incomplétude et de l'inconsistance partielle, et des théories telles que les logiques non monotones, les logiques modales, les théories des probabilités bayesiennes et non bayesiennes, la théorie des fonctions de croyance, les ensembles flous, la théorie des possibilités, la théorie de l'évidence, ou encore les réseaux de croyance ${ }^{6}$. Dans cette perspective, la logique floue contribuerait à aborder des problèmes de manipulation de connaissances imparfaites qui ne pouvaient pas être posés en théorie des probabilités. De plus, le développement de la logique floue constituerait un événement historique significatif, dans la construction d'un pont privilégié et particulièrement fécond entre le champ de la logique et celui des connaissances imparfaites.

Un troisième registre historiographique, d'inspiration plus logicienne, met avant tout l'accent sur les problèmes et les limites rencontrées par la logique binaire face aux paradoxes logiques classiques. Ces narrations présentent alors l'émergence de la logique floue comme une solution à ces problèmes et un dépassement de ces limites. L'histoire de ces problèmes est alors renvoyée à un passé bien plus ancien.

Ainsi Bart Kosko, professeur à l'université de Californie du Sud à Los Angeles et figure renommée de la logique floue, place l'essor de cette logique dans la continuité des travaux menés par Jan Lukasiewicz sur les logiques à plusieurs valeurs de vérité, ainsi que de la philosophie du vague de Bertrand Russell ${ }^{7}$. Selon lui, l'essor de la logique floue constitue une réponse à «l'échec de la logique classique », et à «l'échec " de la loi du tiers exclu d'Aristote face aux paradoxes grecs, soulignés et développés par Russell. Selon Kosko, le philosophe de la mécanique quantique Max Black dessina les premiers diagrammes flous, dans le prolongement du développement par Lukasiewicz d'une logique à plusieurs valeurs de vérité dans les années $1920^{8}$. En nommant ces ensembles des ensembles vagues,

6. Voir, en part., Dubors, Prade et Smets, 1994; Smets, 1991.

7. KosKo et IsAKa, 1993.

8. BLACK, 1937. 
Black aurait prolongé les travaux de Russell. Trente ans plus tard, Zadeh appliquait la logique de Lukasiewicz à chaque élément d'un ensemble, créant ainsi une algèbre complète pour les ensembles flous.

Un quatrième registre historiographique fait remonter l'histoire de la logique floue à l'Antiquité grecque. Pour donner une illustration de ce type de narration, on peut par exemple se pencher sur un exposé de présentation de la logique floue élaboré pour un séminaire de formation continue pour ingénieurs aux États-Unis en 1992. James Brule, l'auteur de ce texte, y construit une généalogie des idées liant Héraclite et Platon à Zadeh, en passant par Hegel, Marx, Engels et Donald Knuth ${ }^{9}$. Il s'agit là d'un exposé succinct, sans référence précise aux travaux des auteurs auxquels il est fait allusion. Pour Brule, quand Parménide proposa la première version de la loi du tiers exclu, affirmant que toute proposition est soit vraie, soit fausse, des objections fortes et immédiates s'élevèrent, comme celle d'Héraclite qui avança l'idée que certaines propositions pouvaient être à la fois vraies et non vraies. Platon fait alors figure de fondateur de ce qui allait devenir la logique floue, dans la mesure où il indiquait qu'il existait un domaine tiers (par-delà le vrai et le faux) où les opposés « prenaient certaines libertés ». Pour Brule, c'est dans cet esprit que Zadeh aurait élaboré la logique floue comme une logique possédant une infinité de valeurs de vérité, sur les traces successives de Hegel, de Marx, d'Engels et, plus récemment, du travail de Lukasiewicz et de Knuth sur les logiques à trois valeurs de vérité.

Mais l'histoire des idées n'a pas l'exclusive dans cette historiographie. Plusieurs narrations essaient de situer l'invention de la logique floue dans le cadre d'une histoire événementielle, à l'appui de quelques données institutionnelles, techniques et industrielles. Analytiquement peu élaborés, ces récits ne prétendent pas offrir plus que quelques repères. lls mettent en scène non seulement d'autres acteurs que Zadeh, mais également d'autres ressources, comme des dispositifs technologiques, des produits commerciaux, des laboratoires, des associations, des colloques, des revues, ou encore des articles jugés clefs ou estimés avoir fait date. L'introduction de ces données contribue à faire apparaître l'essor de la logique floue comme la résultante d'une action collective ${ }^{10}$, et non plus simplement comme un pur produit de l'esprit de Zadeh. Simultanément, l'objet " logique floue " n'est plus idéalisé, il est littéralement excorporé, désenclavé du cerveau de son inventeur. La description suivante, rédigée pour le compte d'une

9. Archives du forum électronique d'Internet « comp.ai.fuzzy $»$, article $n^{\circ} 40$.

10. Voir, également, Gaines et KoHOut, 1977. Cet article présente le développement de la logique floue comme le produit d'une action collective impliquant un grand nombre d'acteurs, en présentant une large bibliographie sur la logique floue et des travaux jugés connexes jusqu'au milieu des années 1970. 
société japonaise d'électronique mettant en avant l'utilisation de la logique floue dans ses produits, illustrera clairement ce point ${ }^{11}$ :

"The year 1990 witnessed the 25th anniversary of the invention of Fuzzy theory. It has undergone numerous transformations since its inception with a variety of Fuzzy Logic applications emerging in many industrial areas. Dividing these past years into different stages, the early 1970s are the "theoretical study" stage, the period from the late 1970s to early 1980s the stage of "developing applications for control", and that from late 1980s to the present the stage of "expanding practical applications". Here are the major events in the history of Fuzzy Logic : 1965 : Professor L. A. Zadeh of the University of California at Berkeley introduces "Fuzzy sets" theory. 1968 : Zadeh presents "Fuzzy algorithm". 1972 : Japan Fuzzy Systems Research Foundation founded (later becoming the Japan Office of the International Fuzzy Systems Association (IFSA)). 1973 : Zadeh introduces a methodology for describing systems using language that incorporates fuzziness. 1974 : Dr. Mamdani of the University of London, UK succeeds with an experimental Fuzzy control for a steam engine. $1980: F$. L. Smidth \& Co. A/S, Denmark, implements Fuzzy theory in cement kiln control (the world's first practical implementation of Fuzzy theory). 1983 : Fuji Electric Co., Ltd. implements Fuzzy theory in the control of chemical injection for water purification plants (Japan's first). 1984 : International Fuzzy Systems Association (IFSA) founded. 1985: 1st IFSA International Conference. 1987 : 2nd IFSA International Conference. (Exhibit of OMRON's Fuzzy controller, a joint development with Assistant Professor Yamakawa). Fuzzy Logic-controlled subway system starts operation in Sendai, Japan. 1988 : International Workshop on applications of Fuzzy Logic - based systems (with eight Fuzzy models on display). 1989 : The Laboratory for International Fuzzy Engineering Research (LIFE) established as a joint affaire between the Japanese Government, academic institutes and private concerns. Japan Society for Fuzzy Theory and Systems founded. [...] 1987 marked the start of Japan's so-called "Fuzzy boom", reaching a peak in 1990. A wide variety of new consumer products since then have included the word "Fuzzy" on their labels and have been advertised as offering the ultimate in convenience. »

Bien que sommaire du point de vue de la construction analytique, omettant notamment toute discussion de nature épistémologique, une telle description suggère toutefois clairement que le développement de la logique floue, loin de consister en un simple déploiement d'idées, met en jeu tout un ensemble d'acteurs, de ressources variées, et de liens unissant (à un certain niveau descriptif) science, technologie et société. Cette image est conforme à celles proposées dans des narrations, qui pour rendre compte de l'essor de la logique floue depuis le milieu des années 1960, insistent sur

11. Archives du forum électronique d'Internet « comp.ai.fuzzy », article $n^{\circ} 124$. 
de nombreux points tels que : l'implication de plusieurs milliers de chercheurs et d'ingénieurs à travers le monde (en particulier, aux États-Unis, en Europe de l'Ouest, au Japon, en Chine); la réalisation de travaux dans une multitude de disciplines et de champs de recherche (notamment logique, philosophie, mathématiques, automatique, informatique, linguistique, psychologie, sciences sociales, physique, biologie, médecine, théorie des jeux, reconnaissance des formes, gestion des bases de données, intelligence artificielle); l'implication d'un grand nombre de sociétés industrielles développant des produits informatiques et électroménagers dits flous; une production textuelle considérable, incluant livres, articles de revues, rapports d'experts, articles de journaux ${ }^{12}$.

MÉDIATIONS MATÉRIELLES ET PRATIQUES DE DÉMONSTRATION

L'historiographie actuelle sur la logique floue apparaît donc osciller entre l'oubli des conditions institutionnelles, techniques et industrielles de son essor, et l'omission de ses développements que l'on pourrait qualifier de théoriques et d'épistémologiques. On voit par là même que l'exploration de l'histoire de la logique floue appelle une méthodologie permettant de prendre en compte l'ensemble de ces données, d'en proposer un traitement homogène, et de combler ainsi le fossé qui sépare les deux grands registres narratifs précédents.

Si le déploiement de ressources hétéroclites que nous avons souligné apparaît irréductible au point de focalisation d'une histoire des idées (à savoir à un simple engagement d'idées), il n'est guère plus réductible à l'objet d'une simple histoire disciplinaire, puisque les contributions à la logique floue ne peuvent être uniquement associées à une ni même à plusieurs disciplines scientifiques. Compte tenu des descriptions précédentes, l'écriture de l'histoire de la logique floue semble exiger bien plus, la saisie de l'émergence d'un objet impliquant tout à la fois science, technologie et société ${ }^{13}$.

Une voie de recherche qui se dessine alors consiste à tenter de mettre en évidence les médiations entre développements théoriques, techniques, industriels et institutionnels de la logique floue. Autrement dit, il s'agit de

12. Archives du forum électronique d'Internet «comp.ai.fuzzy ", en part., les articles $\mathrm{n}^{\mathrm{os}}$ 124, 1555, 2072; Synthèse Technologies de l'information, 1989-1990; La Lettre du club logique floue, 1991.

13. Voir BriaN, 1994 : Éric Brian formule et traite un problème similaire, lié à l'émergence de la statistique au xvin' siècle. 
chercher à saisir dans un même mouvement l'implication et la production d'un nouveau formalisme et de formes d'ordre technologique et social ${ }^{14}$, ainsi que les liens éventuels qui unissent ces éléments.

Il convient donc, en premier lieu, de suivre et d'analyser en détail le travail des acteurs de la logique floue, en identifiant ses conditions matérielles et ses espaces de réalisation ${ }^{15}$. De ce point de vue, les pratiques savantes d'écriture et de lecture ne sauraient être ignorées, ainsi qu'Imre Lakatos, notamment, l'avait envisagé pour l'histoire de la logique lorsqu'il notait : «Malheureusement, même les meilleurs historiens de la logique tendent à ne prêter attention qu'aux changements au niveau de la théorie logique sans remarquer leurs liens avec les changements au niveau de la pratique logique ${ }^{16}$.»

Il faut cependant insister sur le fait qu'en prenant l'activité d'écriture et de lecture des chercheurs pour objet, il ne s'agit pas simplement de faire apparaître les processus de production d'abstraction dans leur matérialité ${ }^{17}$, mais bien surtout de tenter de saisir dans un même mouvement comment des représentations théoriques et des formes d'ordre social et technologique sont produites ou transformées.

Au reste, le travail de Jack Goody sur la raison graphique illustre bien l'intérêt d'une telle démarche sur des terrains déconnectés des espaces de production logique ou mathématique ${ }^{18}$. Au lieu d'appréhender le travail d'écriture comme un acte immatériel de représentation qui «s'extrairait » du social, Goody montre en effet comment la matière même d'une opération de transcription est susceptible tout à la fois de modifier des dynamiques de relations sociales, et de transfigurer des systèmes de représentations du monde.

Toutefois, prendre uniquement pour objet ces pratiques d'écriture et de lecture apparaît fort limitatif à propos d'une période (en l'occurrence, le dernier tiers du $\mathrm{xx}^{\mathrm{e}}$ siècle) où le rapport de la production d'abstractions à ses conditions matérielles semble avoir fortement évolué, compte tenu notamment de l'essor de l'informatique.

14. Nous employons ici le terme « social » dans un sens tout à fait fondamental, analogue à celui que Durkheim met en jeu lorsqu'il se demande pourquoi la " vie sociale " est " source de vie logique », et qu'il met en doute l'idée selon laquelle les hommes se sont associés pour satisfaire à de simples besoins spéculatifs. Voir DurKHEIM, 1990, p. 616-617.

15. Voir, à ce titre, la méthodologie proposée pour une histoire matérielle de l'abstraction, in BrIAN, 1995.

16. Lakatos, 1984, p. 104.

17. À ce sujet, voir Alker, 1982, p. 29 : "There is a lot to be said for treating and teaching the Frege-Russell program of axiomatic, anti-metaphysical formal, extensional logic as an extraordinarily dialectical, anti-Hegelian exercise, in some ways parallel to Marx and Lenin's efforts to recast Hegelian thought in a materialist vein. "

18. GoodY, 1977. 
Lorsque nous avons effectué des observations empiriques sur le travail des chercheurs en logique floue en France et aux États-Unis au début des années 1990, nous avons été mis en présence d'acteurs qui non seulement produisaient des inscriptions logiques sur des feuilles de papier, mais aussi exhibaient et discutaient les propriétés de la logique floue à partir de logiciels informatiques ou d'automatismes qu'ils avaient conçus, et dont ils commentaient le fonctionnement face à des pairs ou à des industriels. Dans ces conditions, le travail démonstratif pour les protagonistes revêtait des formes et des enjeux divers ${ }^{19}$. Cet exercice pouvait, en effet, prendre la forme d'une rédaction d'article, d'un exposé oral et au tableau de résultats lors d'un séminaire, d'une présentation du fonctionnement d'un dispositif technique fonctionnant «à l'aide » de la logique floue. La pratique des démonstrations pouvait engager la construction de nouveaux objets formels, l'adhésion de lecteurs ou d'auditeurs à certains résultats, la reconnaissance d'un travail, des chaînes de crédit et, plus ou moins directement, des formes de financement d'une activité de recherche, le développement et la vente de produits.

Les différentes formes de démonstration engagées et leurs usages sociaux apparaissaient ainsi comme des médiations cruciales du codéveloppement théorique, technique, industriel et institutionnel de la logique floue. Tel est le point qui sera développé dans ce qui suit. La période allant du début à la fin de la première moitié des années 1990 sera plus particulièrement étudiée, pour pouvoir bien mettre en évidence la diversité des pratiques démonstratives et de leurs enjeux pour l'essor de la logique floue, à partir des observations empiriques qu'il a été possible de réaliser. L'analyse portera sur des médiations tout à fait privilégiées dont la signification et la portée seront précisées au fil de notre développement $:$ il s'agira des ressources mobilisées au cours des actes de lecture et d'écriture, de certains outils de visibilité de définitions concurrentes de la logique floue et, enfin, des « démos » de dispositifs techniques.

Un passage d'un article rédigé par une figure de la logique floue déjà mentionnée et citée, Bart Kosko, sera tout d'abord étudié. L'importance de ce texte et la pertinence de ce choix par rapport à l'histoire récente de la logique floue apparaîtront clairement a posteriori.

RESSOURCES POUR LES PRATIQUES D'ÉCRITURE ET DE LECTURE

Dans un article intitulé «Le flou contre les probabilités», son auteur, Kosko, tente de démontrer la propriété de la logique floue de résoudre le

19. Rosental, 1996. 
paradoxe du barbier formulé par Russell. Ce texte pourrait être examiné comme un support d'idées, dont on pourrait faire abstraction pour établir, par exemple, une généalogie de concepts propres à la logique floue. Mais il sera considéré différemment, c'est-à-dire comme un dispositif scriptural susceptible de produire des effets propres sur ses lecteurs, une fois confronté à des pratiques de lecture dont il convient de souligner les conditions particulières. L'étude portera sur l'objet logique floue situé historiquement qui émerge de ce texte, au fil des associations particulières et de la construction d'un champ de concurrence qu'il contribue à définir. La démonstration en question est reproduite ci-après ${ }^{20}$ :

« Le barbier de Russell est un homme barbu qui vit dans une ville où il rase un homme si et seulement si ce dernier ne se rase pas lui-même. Donc qui rase le barbier? S'il se rase lui-même, alors par définition il ne se rase pas. Mais s'il ne se rase pas lui-même, alors par définition il se rase.

[1] Donc il se rase et ne se rase pas - contradiction (« paradoxe »). Gaines ${ }^{21}$ a noté que ce cas paradoxal peut être interprété numériquement de la façon suivante.

[2] Soit $\mathbf{S}$ la proposition exprimant que le barbier se rase, et non-S qu'il ne se rase pas. Alors $S$ implique non-S et non-S implique $S$, les deux propositions sont logiquement équivalentes :

[3] $S \Leftrightarrow$ non-S

[4] Des propositions équivalentes ont les mêmes valeurs de vérité :

[5] $\mathrm{V}(\mathrm{S})=\mathrm{V}($ non-S $)$

[6] $V(S)=1-V(S)$

La résolution donne pour $\mathrm{V}(\mathrm{S})$ le milieu de l'intervalle de vérité [...] :

[7] $\mathrm{V}(\mathrm{S})=1 / 2$.

[...] En logique bivalente, les deux énoncés $S$ et non-S doivent avoir la valeur de vérité 0 ou 1 . La résolution floue du paradoxe utilise uniquement le fait que les valeurs de vérité sont égales. [...] La valeur médiane 1/2 émerge de la structure du problème."

Partant de ce qui est parfois considéré par ses contemporains comme un des paradoxes dont la formulation a marqué la fin du logicisme au début du $\mathrm{xx}^{\mathrm{e}}$ siècle ${ }^{22}$, Kosko tente ici de montrer que la logique floue, contrairement

20. Notre traduction de Kosko, 1990, p. 219-220. La numérotation entre crochets des énoncés relève de notre propre initiative.

21. Gaines, 1983.

22. Voir, notamment, LARGEAULT, 1993, p. 3-24. Le terme de logicisme fait généralement référence au programme de Frege-Russell de fondement d'une logique unitaire et totale, sousjacente à toute la réalité. Il s'oppose notamment à des constructions formalistes ou algébristes de la logique, sous la forme de modèles éclatés avec des domaines et des relations différents. Pour une discussion de l'importance relative du paradoxe du barbier par rapport à d'autres paradoxes, et pour une discussion des similarités et des différences, voir SAINSBURY, 1988. Pour une autre approche des paradoxes du barbier et du menteur, voir BARWISE et ETCHEMENDY, 1987. 
à la logique binaire, n'est pas victime de contradictions. Comme il l'explique en effet un peu plus loin dans son article, le terme " paradoxe » n'est selon lui qu'un euphémisme utilisé pour éviter d'admettre les contradictions de la logique binaire.

Dans cette perspective, l'auteur tente de montrer que le fait de disposer de valeurs de vérité comprises entre 0 et 1 , en d'autres termes d'énoncés ni complètement vrais ni complètement faux, ressources qu'il associe de fait à la logique floue, confère la propriété de résoudre le paradoxe du barbier. Comment faut-il ici entendre le terme " résoudre "? Ou pour être plus précis, quelles ressources et quelles pratiques correspondent à ce terme?

La première étape de cette résolution consiste à formuler un petit scénario, un énoncé en prose destiné à introduire une manipulation spécifique d'inscriptions. En termes wittgensteiniens, il s'agit d'amener et de justifier des opérations graphiques visualisables sur le papier par l'exhibition de formes de vie qui les naturalisent ${ }^{23}$. Le petit scénario exprimé en prose sur le barbier met précisément en scène une forme de vie qui, suivie de traductions adéquates, doit rendre «naturelle »le recours à une valeur de vérité intermédiaire entre 0 et 1 (ce que l'auteur exprime en affirmant que cette valeur de vérité émerge de la "structure " même du problème).

Cette première étape est alors suivie d'opérations de traduction, l'auteur cherchant à faire admettre l'équivalence entre toute une série d'énoncés qui mobilisent divers graphismes. Kosko s'exprime par périphrases successives, afin d'amener et de faire apparaitre des termes classiquement consacrés en symboles par des graphismes spécifiques. Cette démarche démonstrative d'ensemble (impliquant l'élaboration d'un scénario, des traductions, une production de périphrases et de symboles graphiques) relève dans ses modalités de pratiques tacites fortement sédimentées ${ }^{24}$.

C'est ainsi qu'à la proposition [1], Kosko lui substitue la périphrase [2]. Cette périphrase présentée comme équivalente, c'est-à-dire substituable visuellement à l'inscription précédente, ou en d'autres termes ce détour de formulation, lui permet de préparer littéralement graphiquement une symbolisation ou une formalisation en [3], à savoir une mise en symboles ou une mise en formes matérielles nouvelles (en juxtaposant les termes «formes » et «matérielles», on insiste sur le fait qu'il s'agit avant tout

23. Ludwig Wittgenstein suggère, par ex., que l'opération qui consiste à prendre la racine du nombre « moins un " a pu devenir acceptable en lui associant une traduction graphique de manipulation de vecteurs, visualisable sur le papier, voir WITTGENSTEIN, 1976, p. 226 : « Similarly, by surrounding $\sqrt{-1}$ by talk about vectors, it sounds quite natural to talk of a thing whose square is -1 . That which at first seemed out of the question, if you surround it by the right kind of intermediate cases, becomes the most natural thing possible. "

24. La sédimentation de telles pratiques peut être retracée dans l'enseignement de la logique tel qu'il est pratiqué aux États-Unis depuis la fin de la Seconde Guerre mondiale. À ce sujet, voir Rosental, 1993. 
d'une production de nouveaux graphismes). Substituant alors à la proposition [3] la traduction à l'aide de graphismes consacrés [5], en introduisant les inscriptions correspondant à une " hypothèse " usuelle, c'est-à-dire à une pratique logique très répandue [4], Kosko parvient donc à traduire, au fil de cette production d'inscriptions, le scénario initial en un " calcul ", un ensemble spécifique de manipulations. En imposant, en effet, implicitement la manipulation selon laquelle « $\mathrm{V}($ non-S) $=1-\mathrm{V}(\mathrm{S})$ », l'auteur aboutit à une équation algébrique simple [6], qu'il résout en [7] en mobilisant tacitement des pratiques de calcul élémentaires.

Il est donc clair que l'apparition graphique de la capacité particulière de la logique floue à résoudre le paradoxe du barbier relève non seulement de la mobilisation de pratiques tacites gouvernant le déroulement d'ensemble et de détail de la production d'inscriptions, mais aussi de ressources pour l'écriture et la lecture imprimant si l'on peut dire leur propre logique ${ }^{25}$, des traces matérielles consultables et reconsultables ponctuant le déplacement des regards des lecteurs ${ }^{26}$. Ce processus échappe par là même à la mise en œuvre d'un simple conventionnalisme ${ }^{27}$, et n'est pas non plus justiciable d'un relativisme social qui ne porterait aucune attention aux manipulations d'inscriptions jugées arbitraires, ou dont l'action serait perçue comme entièrement déterminée par des relations d'autorité ${ }^{28}$. L'action démonstrative (que ce soit l'action d'écriture ou de lecture de la démonstration depuis un poste de travail), loin d'être elle-mềme abstraite ou localisable simplement dans l'esprit des acteurs, ou simplement assignable à des pratiques contextuelles de communautés scientifiques plus ou moins vastes, est ici au contraire distribuée jusque dans les inscriptions matérielles ${ }^{29}$.

On observe ainsi comment un dispositif nécessaire à des pratiques d'écriture et de lecture, confronté à des usages adéquats, pouvait contribuer au début des années 1990 à la mise en avant d'un système de représentation

25. Voir, à nouveau, le travail de Goody, 1977, sur la raison graphique. Lue elle-même dans une perspective matérialiste minimale, voir aussi, à ce sujet, la lecture par Jacques Bouveresse de Wittgenstein, in Bouveresse, 1976, p. 97 : « Le fait qu'elle [une proposition] soit composée d'éléments en tel ou tel nombre, se rapportant les uns aux autres de telle manière, lui confere une potentialité interne de signification, autrement dit d'être affectée à la représentation d'un certain type de situation. "

26. Voir, également, Livingston, 1985.

27. Voir, aussi, la tentative d'Andrew Pickering et d'Adam Stephanides d'échapper au conventionnalisme, en distinguant mouvements libres et mouvements forcés dans le travail de recherche sur les quaternions du mathématicien Hamilton, in Pickering et Stephanides, 1992. En revanche, pour une sociologie dénonciatrice développée à partir du cas d'Hamilton, voir BLOOR, 1981.

28. En part., ce type d'objet échappe complètement au relativisme social de Harry Collins, qui écrit pour sa part, in CoLuns, 1985, p. 159 : " The locus of knowledge is not the written word or symbol but the community of expert practitioners."

29. Voir, aussi, le travail sur la cognition distribuée, liée à la manipulation de formalismes, réalisé in Hưtchins, 1995. 
particulier. Avant d'examiner comment ce même dispositif, relayé par d'autres, contribuait à créer des associations et des oppositions entre acteurs, importantes du point de vue du développement de la logique floue, il convient de mieux cerner l'extension du système de représentation ainsi défini. Il serait possible, en effet, de penser avoir affaire ici à l'élaboration graphique d'une propriété formelle pure. Si l'on veut saisir toutes les dimensions du travail démonstratif effectué dans cet article, il est important de noter qu'il n'en est rien.

Dans l'enchaînement immédiat de cette démonstration, Kosko met en effet en avant des propriétés historiques, culturelles et réalistes de la logique floue en liaison avec l'histoire particulière du paradoxe du barbier. Toujours par un procédé d'écriture, qui mêle les registres démonstratifs, l'auteur affirme dans la suite de son article qu'il a ainsi démontré que la logique floue est la seule description réaliste du monde (les énoncés du monde ne sont pas tous complètement vrais ou faux). Le paradoxe du barbier témoigne selon lui incontestablement de l'inconsistance de la logique binaire et notamment du principe du tiers exclu (selon lequel une proposition est soit vraie soit fausse). En rationaliste, Kosko estime avoir démontré que les résistances à l'abandon de la logique binaire et du principe du tiers exclu sont elles-mêmes irrationnelles, et relèvent par suite d'explications historico-culturelles. Pour l'historien Kosko, la " foi " dans la logique binaire et dans le principe de contradiction relève d'une prédilection culturelle transmise en Occident dans une tradition d'enseignement qui remonterait « au moins » à Aristote. Selon lui, la logique floue marque un tournant entre la fin de la pensée binaire occidentale et la prédominance à venir de la pensée et de la civilisation orientales.

La démonstration de la propriété formelle de la logique floue à résoudre le paradoxe du barbier est ainsi elle-même le point de départ d'une démonstration mêlant des registres divers pour mettre en avant, également de façon textuelle, des propriétés réalistes, historiques et culturelles de cette logique. Si plusieurs travaux en sociologie des sciences ont montré comment des scientifiques peuvent chercher à reconstruire le monde à partir de leur laboratoire ${ }^{30}$, nous voyons ici comment un logicien, par la médiation de l'écriture d'un simple article, et partant du déploiement graphique d'une propriété logique formelle, tente de reconstruire des catégories « universelles » de la représentation et l'histoire des civilisations.

Écrire l'histoire de la logique, ou même des civilisations, peut être en fait appréhendé comme un moyen à part entière pour les spécialistes de la logique floue de démontrer la valeur de cette logique, et du travail effectué dans ce domaine. Cela est bien entendu valable pour les histoires de la

30. Voir, en part., Latour, 1983. 
logique floue qui ont été examinées au début de cet article. En d'autres termes, les histoires de la logique floue qui viennent d'être analysées peuvent être perçues rétrospectivement comme d'authentiques démonstrations. Même si on ne peut pas les réduire à cette unique dimension, les histoires qui situent l'essor de la logique floue essentiellement entre le milieu des années 1960 et les années 1990 constituent un moyen de promouvoir le rôle de chercheurs contemporains, en insistant sur un essor ex nihilo de la logique floue au travers de leurs actions singulières. À l'inverse, les récits qui situent l'origine de la logique floue dans des traditions plus anciennes correspondent généralement à des stratégies de légitimation.

Cependant, après avoir observé comment la construction d'une opposition historique entre systèmes logiques et la constitution graphique de liens entre science et société contribuent en tant que telles, dans l'article de Kosko, à mettre en avant une définition de la logique floue, plusieurs questions se posent. Quelles associations et oppositions cette définition engendre-t-elle? Quels en sont les autres véhicules, quels obstacles rencontre-t-elle, et en quoi marque-t-elle éventuellement l'histoire de la logique floue au début des années 1990 ? Il convient maintenant, comme annoncé, d'examiner un certain nombre d'autres médiations, qui prennent pour ainsi dire le relais de l'article de Kosko pour véhiculer plus avant l'ensemble de propriétés précédemment définies pour la logique floue ${ }^{31}$. On l'aura compris, l'étude des formes de démonstration rejoint très étroitement à ce stade la question des outils de visibilité des propriétés attribuées à la logique floue.

VISIBILITÉ

Au début des années 1990, un des ouvrages de Kosko ${ }^{32}$ avait obtenu un succès considérable d'édition, devenant par là même un véhicule majeur de la définition de la logique floue. Ce livre, qui mettait en avant les mêmes propriétés pour cette logique que celles qui viennent d'être examinées, était en effet utilisé comme manuel d'enseignement dans les départements d'informatique et de sciences de l'ingénieur de nombreuses universités américaines. D'après des témoignages convergents, ce manuel avait été plus vendu aux États-Unis que l'ensemble des autres ouvrages dédiés à la

31. Il faut noter qu'une analyse sémiotique du texte de Kosko, se focalisant sur ce seul texte, trouverait ici ses limites. Pour un exemple d'approche sémiotique des mathématiques, voir ROTMAN, 1993.

32. Kosko, 1992. 
logique floue, faisant de Kosko une des figures centrales de ce domaine. Deux autres véhicules importants assurant le déploiement de ces propriétés consistaient dans un livre de vulgarisation du même auteur ${ }^{33}$, et dans une publication destinée au grand public, rédigée par d'autres, qui reprenait ses thèses ${ }^{34}$. À cela s'ajoutait tout un ensemble d'autres médiations: des articles comme celui que nous avons examiné, des cours à l'université où Kosko enseignait (University of Southern California à Los Angeles), et toujours aux États-Unis des conférences, des séminaires de formation continue, des séances de dédicace dans des librairies, des interventions à la radio, des entretiens accordés aux journalistes cités dans des articles de presse, des campagnes de promotion de ses écrits dans des forums électroniques, dans des revues spécialisés ou des journaux d'associations d'ingénieurs, etc. Bref, tout un ensemble de médiations qui n'étaient pas propres à la mise en avant d'objets logiques et que l'on retrouvait dans d'autres modes de vie universitaire où les pratiques de l'écrit étaient essentielles.

La définition mise en avant par Kosko pour la logique floue suscitait en fait tout autant des adhésions que des oppositions parmi les spécialistes de ce domaine. Des coalitions se formaient vis-à-vis de cette définition (en sa faveur ou à son encontre). Ainsi, face aux modes de déploiement et de reprise toujours plus nombreux des propriétés précédemment décrites de la logique floue, et à la visibilité grandissante de la définition qui lui correspondait, de nombreux chercheurs se coalisaient pour avancer une définition concurrente de cet objet aussi visible que possible. En fait, il est possible d'appréhender le terme « logique floue » moins comme un emblème durkheimien que comme un « énoncé collectif », pour reprendre l'expression de l'historien Alain Boureau ${ }^{35}$. L'expression " logique floue " était, en effet, adoptée par des chercheurs mettant en avant des définitions contradictoires de cette logique. L'utilisation commune de cette formule liait différents objets et l'action de divers chercheurs d'une façon souple et complexe. Si les acteurs s'appropriaient cet énoncé de façon quasi singulière pour qualifier des objets en fait différents, il n'en demeure pas moins que l'existence d'une terminologie commune jouait en tant que telle un rôle dans le détail et la globalité du développement de cette logique.

Les espaces où plusieurs définitions de la logique floue s'affrontaient, et où par ailleurs des chercheurs développant d'autres approches manifes-

33. Kosko, 1993.

34. McNeill et Freiberger, 1992.

35. Boureau, 1989, et Boureau, 1992, p. 1072 : «J'appelle un énoncé collectif [...] un fragment verbal (ou iconique), qui crée autour de lui une certaine convergence des langages et des pensées, par le jeu d'un flou structurel susceptible de capter une thématique encore implicite et d'accueillir les projections et les appropriations les plus diverses. "Voir, aussi, in JACQ, 1996, l'utilisation de la notion d'énoncé collectif pour rendre compte de la construction et du rôle de l'énoncé «politique scientifique " en France après la Seconde Guerre mondiale. 
taient leur hostilité à la logique floue, étaient nombreux. Cette lutte s'engageait notamment dans le cadre de l'introduction d'enseignements universitaires, d'associations, de séminaires de formation continue, de l'organisation de conférences, de la passation de contrats industriels, de processus de recrutement, de soutenances de thèse, d'articles de revue ou de presse, ou encore de la construction de récits historiques sur la logique floue. Il n'est pas ici possible d'examiner systématiquement toutes ces données et d'établir une cartographie quelque peu fine des définitions émergentes pour la logique floue et des associations d'acteurs correspondantes. Il est, en revanche, possible d'illustrer notre propos en évoquant quelques exemples.

Lors d'un entretien que nous avons réalisé au début de l'année 1994, un spécialiste réputé de la logique floue ${ }^{36}$ estimait que Willard Van Orman Quine avait bien montré que le paradoxe du barbier ne pouvait pas être résolu à l'aide d'une logique à plusieurs valeurs de vérité ${ }^{37}$. Pour ce chercheur, il y avait un « truc » dans les traductions effectuées par Kosko, et la logique floue ne possédait pas cette capacité formelle de résolution. Les propriétés historiques et culturelles attribuées par Kosko à la logique floue étaient à ses yeux extravagantes, tout autant que ses thèses d'histoire des sciences conduisant au dénigrement de la tradition aristotélicienne, dans la mesure où les logiques à plusieurs valeurs de vérité avaient été développées selon lui dans cette tradition.

Par-delà la médiation de la dénonciation orale effectuée au cours de cet entretien pour véhiculer une autre définition de la logique floue, ce chercheur menait d'autres actions. Il venait de publier une critique virulente d'un ouvrage grand public reprenant les thèses koskoiennes. Il participait également à la rédaction d'un manuel d'enseignement concurrent de celui de Kosko où, dans le corps du premier chapitre, il proposait comme exercice de démontrer que la solution de Kosko du paradoxe du barbier était fausse. Les médiations de cette déconstruction (encore une fois graphique) consistaient à repérer et à mettre en cause une des traductions, et à lui substituer à cet embranchement une nouvelle série de traductions conduisant à nouveau à un paradoxe ${ }^{38}$.

\footnotetext{
36. Nous préserverons son anonymat.

37. QuiNe, 1976.

38. Les processus que nous décrivons de transformation et de déplacement visuels se distinguent de ce que Kuhn qualifie de « révolutions comme transformations dans la vision du monde ", ne serait-ce que parce que nous ne mettons pas en jeu de modification de paradigme. Dans notre perspective, si des modifications de la vision du monde pour des groupes d'acteurs relativement larges s'opèrent, c'est dans l'enchaînement de médiations qui peuvent intervenir y compris à un niveau interactionniste. L'analyse de la diversité de ces médiations ne saurait cependant être ramenée à des thèses générales de psychologie cognitive (notamment de psychologie de la forme) qui constituent le point de départ de l'analyse kuhnienne. Voir KUHN, 1983, p. 157-188.
} 
Comme autre exemple de médiations déployées dans de telles tensions, on peut noter que dans la première moitié des années 1990, les organisateurs d'une grande conférence sur la logique floue ${ }^{39}$ estimaient que les thèses de Kosko étaient erronées, donnaient une image fausse de la logique floue et menaçaient sa crédibilité en gagnant en visibilité. Aussi avaient-ils essayé de minimiser la visibilité de Kosko à cette conférence en lui attribuant un thème de communication qui ne touchait pas de façon centrale à la définition de la logique floue. Dans les couloirs de la conférence, ils exprimaient par ailleurs leurs craintes à d'autres universitaires, les encourageaient à évoquer ce problème et à dénoncer ouvertement les erreurs effectuées par Kosko lors de ses interventions. Ils insistaient sur le fait que les thèses de Kosko menaçaient l'ensemble de l'activité de recherche dans ce domaine, tout particulièrement dans le contexte d'une "guerre froide» avec les partisans d'autres approches en intelligence artificielle, liée à une concurrence de plus en plus forte pour les mêmes sources de financement. D'ailleurs, un universitaire se présentant comme un partisan de l'intelligence artificielle « classique " avait publié un article en 1993, où il tentait de démontrer certaines contradictions de la logique floue, partant précisément de la présentation de cette logique effectuée dans le texte de Kosko que nous avons analysé. Cet article avait été primé par les organisateurs de la grande conférence américaine sur l'intelligence artificielle «AAAI " en 1993, suscitant par la suite une grande controverse aux États-Unis sur cette logique et sur la validité de la démonstration engagée ${ }^{40}$.

S'il n'est pas indispensable d'entrer ici dans les détails de ces débats, on peut en revanche se demander à ce stade pourquoi la question du positionnement de la logique floue par rapport à d'autres approches de l'intelligence artificielle et à leur financement pouvait sembler si importante pour les acteurs. Le lecteur aura d'ailleurs peut-être été intrigué par la métaphore introduite dans notre toute première citation, selon laquelle « une multitude d'applications, d'ordinateurs et de produits flous $»$ devraient, dans les prochains siècles, venir «gonfler les voiles de la logique floue». En fait, il convient maintenant d'examiner comment le développement de la logique floue au début des années 1990 était largement obtenu, que ce soit en Europe, aux États-Unis ou au Japon, par l'exhibition de propriétés de ce formalisme dans des dispositifs technologiques. Les systèmes informatiques et les dispositifs de commande automatique de systèmes mécaniques ou électriques constituaient, en effet, des instruments à l'aide desquels de nombreux chercheurs tentaient de mettre en avant la valeur des propriétés

39. La description qui suit est le produit d'observations et d'entretiens que nous avons réalisés à l'occasion de notre participation à cette conférence, que nous ne situerons pas précisément, afin de préserver l'anonymat des protagonistes.

40. Elkan, 1993. 
de la logique floue, comparativement à d'autres logiques (comme la logique booléenne, par exemple). Les spécialistes de la logique floue investissaient ainsi des activités industrielles très diverses ${ }^{41}$. Nous allons nous intéresser dans ce qui suit à un type de médiation privilégié pour l'exhibition de propriétés de la logique floue, les «démos" (abréviation de démonstrations) du fonctionnement de dispositifs techniques.

\section{DÉMOS}

Dans la première moitié des années 1990, l'exhibition de propriétés de la logique floue dans des logiciels et des dispositifs électromécaniques constituait une médiation importante dans la constitution et le maintien de relations de partenariat entre industriels et chercheurs. Elle permettait à ces derniers d'obtenir des contrats, et de créer à court et à moyen terme des réseaux autonomes pour financer leurs recherches. Cet exercice constituait pour eux un moyen de court-circuiter l'appel à des pairs hostiles, développant d'autres approches, et impliqués eux-mêmes dans une rude concurrence pour financer leur propre activité. Certains ténors de la logique floue voyaient dans l'investissement d'activités industrielles très diversifiées et dans la vente de produits sous l'emblème logique floue, un moyen d'assurer à long terme le rayonnement aussi bien de la théorie que des applications de cette logique. De la même façon, l'exhibition de dispositifs technologiques qualifiés de "neuro-flous", sous le double emblème des réseaux de neurones et de la logique floue, constituait au début des années 1990 une stratégie démonstrative en plein essor. Elle procédait à un certain niveau d'une alliance sociotechnique, développée pour faire front et proposer l'alternative la plus visible possible aux méthodes de l'intelligence artificielle dite classique (avec notamment, pour perspective, l'accroissement du volume des financements industriels dans ces domaines).

Les " démos", terme employé par les protagonistes et repris ici pour rendre compte d'un exercice dont la nature ẹt les usages sociaux sont tout à fait singuliers, jouaient un rôle essentiel dans cette démarche ostentatoire. Cette forme de démonstration consistait en un huis clos où un démonstrateur se faisait le porte-parole d'un dispositif, liant son fonctionnement à des

41. Il est intéressant de comparer le cas de la logique floue avec celui de l'essor de l'analyse mathématique, au travers du développement et de l'engagement de machines à calculer dans un grand nombre d'activités économiques en Angleterre à la fin du xix siècle. Voir WARWICK, 1995. 
propriétés générales. Ces propriétés étaient exhibées face à des témoins, dans une sorte de mouvement d'élévation. "Mouvement d'élévation» constitue, en effet, l'expression la plus adaptée pour décrire la façon dont ces propriétés étaient mises en scène, puisqu'elles étaient présentées comme émanant et se détachant de la matérialité et de la spécificité du dispositif $^{42}$.

Tout un ensemble de situations et de postures correspondaient aux démos. Il arrivait que des chercheurs en logique floue tentent par ce moyen d'intéresser des ingénieurs dans un séminaire de formation continue. Ou alors un ingénieur cherchait, en réalisant une démo depuis le stand d'une grande conférence internationale, et en commentant le fonctionnement d'un dispositif technique en mouvement, à convaincre un responsable d'une société industrielle d'acheter un système flou. Les démos étaient communément mobilisées dans des pratiques de vente. Dans ce cas, un produit, une marque, et l'emblème « logique floue » étaient généralement simultanément promus (par exemple, l'appareil photo de la marque $\mathrm{X}$ utilisant la logique floue). Les démos étaient également mobilisées face à des pairs, le recours à cette forme de démonstration académique tendant à se développer de façon importante.

Pour de nombreux partisans de la logique floue, la mise en œuvre de démos entrait dans le cadre d'un apprentissage long des différentes formes de démonstrations à employer en fonction des situations et des interlocuteurs rencontrés. Le directeur d'un laboratoire français, menant des recherches en logique floue, nous décrivait ainsi comment il avait progressivement réalisé l'importance des démos pour séduire des responsables industriels américains ou japonais peu sensibles aux publications. Il avait pour cela embauché un ingénieur pour construire des dispositifs, et il lui avait fallu de longs mois pour mettre au point à la fois les systèmes et les répertoires pour en commenter le fonctionnement. À l'inverse également, un chercheur français en logique floue, travaillant dans le secteur privé, nous faisait part d'une de ses expériences qu'il jugeait caractéristique du goût immodéré pour les mathématiques, désastreux industriellement, de ce qu'il appelait les technocrates français. Comme il ne parvenait pas à convaincre un directeur de la recherche d'un grand groupe industriel français de l'intérêt de la logique floue en décrivant le principe de fonctionnement de dispositifs, il avait évoqué un résultat formel sur la logique floue. Ce registre avait aussitôt suscité l'enthousiasme de son interlocuteur, ce qu'une démo ne parvenait pas à produire. Ces cas en fournissent une bonne illustration, les acteurs de la logique floue étaient les premiers à construire,

42. On rapprochera ces processus «d'apparition » de ceux analysés in DASToN, 1991, et Claverie, 1991. 
de façon plus ou moins élaborée, une approche sociologique des formes de démonstration dans le cadre même de leur activité.

Les démos, nous l'avons dit, consistaient à exhiber un dispositif technique en action devant une petite assemblée de personnes. Le démonstrateur attribuait des propriétés de la logique floue à son fonctionnement en s'en faisant le porte-parole ou encore son représentant, si ce n'est son représentant de commerce. Ce point sera bien illustré au travers du bref compte rendu d'une démo réalisée en France dans le cadre d'une réunion d'association qui avait pour objet la promotion de la logique floue auprès des industriels. Un expert du domaine exhibait à cette occasion les propriétés de cette logique face à une petite assemblée de chercheurs, d'ingénieurs et de responsables de la recherche de grandes entreprises ${ }^{43}$ :

«Il [le démonstrateur] a en particulier illustré l'efficacité et la simplicité de ce type de contrôle dans le cas du pendule inversé (en équilibre sur sa base), et dans un contexte de modifications dynamiques et même aléatoires: verre d'eau rempli en cours d'expérience et même souris vivantes se déplaçant sur un plateau au sommet d'un pendule, sans en rompre l'équilibre, tout ceci sans changer les règles de contrôle. »

L'objectif d'une telle démo est claire : convaincre des spectateurs de la réalité de certaines propriétés de la logique floue, et constituer des témoins ${ }^{44}$ qui puissent ultérieurement attester de la réalité de ces propriétés. Le déroulement de la démo consiste ici dans l'exhibition par le démonstrateur de propriétés telles que "efficacité », « simplicité ", et dans leur attribution à la logique floue. Le démonstrateur se fait le porte-parole du dispositif en mouvement. Pour introduire ces propriétés, un authentique décor est mis en place ${ }^{45}$. Le démonstrateur met en scène les conditions extrêmes et spectaculaires du fonctionnement du dispositif. En omettant de mentionner le rôle de toute autre médiation dans le fonctionnement de l'appareil (parties mécaniques, par exemple), son représentant déploie un répertoire qui attribue son fonctionnement exclusivement à la logique floue. La spécificité de l'ensemble du système disparaît. Apparaissent à la place les propriétés générales de la logique floue exposées par le démonstrateur, qui s'extraient et émanent de la matérialité de l'appareil, et peuvent être perçues comme exportables à d'autres dispositifs.

43. Compte rendu daté du 15 mai 1991 de la réunion tenue le 19 avril 1991 à Paris, Club Crin Logique floue, Paris, Association Écrin, p. 3.

44. Le rôle de la constitution de témoins dans ce type de situation peut être comparé à celui qui est décrit in Shapin et Schaffer, 1985.

45. Les analyses d'Erving Goffman fondées sur des métaphores théâtrales semblent, en fait, particulièrement bien adaptées à la description des diverses formes de démonstration que nous avons identifiées chez les chercheurs en logique au cours de nos enquêtes. Voir GofrMAN, 1971. 
Il faut, en effet, préciser que certains ingénieurs affirmaient que la commande du pendule inversé (c'est-à-dire d'un pendule oscillant à partir de sa base) est emblématique de la commande d'une fusée au décollage. Dans ces circonstances, il est important de maîtriser les oscillations de la fusée, dans la mesure où elle peut se briser en son milieu si elles sont trop intenses. Aussi, un responsable de la recherche de l'industrie aérospatiale pouvait littéralement voir dans une telle démo la maîtrise d'un problème technologique impliquant des milliards d'investissement. Une fois encore dans ce cas, la perception d'une élévation de propriétés par-delà la matérialité d'un dispositif spécifique pouvait avoir lieu.

Cependant, cette démo était également présentée à un plus large public, par la médiation d'un compte rendu écrit expédié par courrier (et par la médiation de notre article, elle est maintenant présentée à de nouveaux lecteurs). Au cours d'une telle opération, le cercle démonstratif était (et est toujours) élargi d'autant.

La mise en œuvre de telles démos a pu être observée dans des configurations variées. Ainsi, un professeur d'automatique au MIT exhibait les propriétés de la logique floue à ses fréquents visiteurs à l'occasion de tours de laboratoire consistant en une série de démos. Ces tours de laboratoire étaient organisés dans leur moindre détail sous une forme stabilisée. Plusieurs dispositifs en mouvement étaient présentés tour à tour. Ces visites de laboratoire étaient tout particulièrement conçues pour les investisseurs industriels qui subventionnaient les projets de recherche du laboratoire ou desquels cet universitaire souhaitait obtenir un nouveau contrat.

Souvent ce professeur se faisait représentant de commerce et se déplaçait aux États-Unis et au Japon pour démarcher de nouveaux investisseurs. II emportait alors avec lui des cassettes vidéos présentant des dispositifs en mouvement selon un scénario élaboré avec la plus grande attention. Cette stratégie démonstrative lui évitait de se déplacer avec des dispositifs encombrants ou fragiles. Elle lui permettait également d'éviter un investissement en temps considérable et le risque d'échec lié aux aléas d'une réplication en tant réel de démos.

Des colloques, destinés à un public de chercheurs et d'industriels, comprenaient quant à eux dans leur programme communications et démos. Tel était ainsi le cas d'un colloque organisé à Paris en mai 1992. Un quota de participants avait été fixé à l'avance pour permettre le déroulement matériel des démos face à de petites assemblées, pour « qu'il n'y ait pas de groupes trop compacts autour des démonstrations » comme le précisait la brochure de présentation. Un compte rendu de cette manifestation (précisément manifestation des propriétés de la logique floue) décrivait son déroulement en ces termes ${ }^{46}$ :

46. La Lettre des clubs Crin, 1992, p. 7. 
"L'intérêt de cette manifestation, c'est que, en plus des communications par les experts [...], on pouvait assister, dans une salle adjacente, à des démonstrations, sur ordinateur, de logiciels basés sur la logique floue. Les démonstrations ont porté sur le freinage des véhicules, la commande numérique, la fusion de données, l'aide à la décision, les applications financières, ou médicales, etc. Autant de domaines variés où les participants ont pu constater de visu, les performances de la logique floue. Des projections de films et des présentations de matériels ont complété le tout. Il reste à mettre en pratique ce potentiel, et pour cela susciter l'intérêt de certains industriels et des autorités gouvernementales. "

Ce compte rendu rédigé par un membre d'une association, dont la vocation était de promouvoir la logique floue auprès des industriels français, montre en raccourci comment la mise en cuvre de démos pouvait entrer dans le cadre d'une large démarche d'intéressement. Le colloque participait à la constitution de porte-parole de la logique floue, en exhibant des chercheurs présentés comme des experts du domaine. Il contribuait simultanément à la mise en avant de propriétés de cette logique, présentées comme incorporées dans des dispositifs, par diverses médiations : appareils en fonctionnement, vidéos de démos, présentations de logiciels sur des écrans d'ordinateurs. La production de témoins, qui verraient in situ les propriétés de la logique floue, procédait ici d'une entreprise explicite. La recherche d'un intéressement différencié d'acteurs engagés dans des activités diverses (banque, médecine, construction automobile, etc.) se combinait ici avec la mise en avant pour l'ensemble des spectateurs de propriétés universelles de cette logique (universelles au sens d'une capacité à être mobilisées dans tout type d'activité).

Des démos étaient donc mobilisées au début des années 1990 pour construire de nouvelles associations avec des acteurs provenant d'horizons de plus en plus étendus, en exhibant un nombre toujours plus grand de propriétés de la logique floue face à de nouvelles assemblées. En d'autres termes, les démos pouvaient être appréhendées comme des médiations privilégiées entrant dans la construction de liens entre science, technologie et société.

Bien sûr, tout comme à l'occasion de l'exhibition de certaines propriêtés formelles de la logique floue, il arrivait que des chercheurs nient la réalité de ces propriétés. Compte tenu de la rude concurrence entre les partisans de plusieurs approches de l'automatique, les exhibitions de propriétés de la logique floue dans des dispositifs étaient parfois déconstruites par plusieurs médiations. Parmi elles, on peut citer simplement un ensemble d'articles issus d'une revue française d'automatique, procès en paternité qui contestent l'attribution du fonctionnement de dispositifs dits flous à la 
logique floue en tant que telle. Un procès en paternité y prenait par exemple la forme suivante : dans un petit article, un automaticien français, de retour d'un voyage au Japon, cherchait à montrer par la médiation de son récit l'absence de recherches sur la logique floue menées dans ce pays, en décrivant les coulisses des tours organisés de laboratoire. Il tentait de montrer que telle caméra vidéo présentée comme incarnant les propriétés générales de la logique floue devait en fait son fonctionnement à d'autres médiations ${ }^{47}$. Une fois encore, la mise en avant de propriétés de la logique floue devait être appréhendée dans le cadre de démonstrations contradictoires constituant ou déconstruisant leur réalité ${ }^{48}$.

En procédant continûment, depuis l'examen de productions historiographiques jusqu'à l'étude de la mise en auvre de démos à vocations académiques et industrielles, il s'agissait ici de mettre en évidence des médiations importantes du codéveloppement théorique, institutionnel, technique et industriel de la logique floue dans la première moitié des années 1990. Analyser de façon homogène, en partant de l'examen de leurs conditions matérielles, des productions textuelles à visée théorique ou historiographique, divers outils de visibilité de définitions concurrentes de la logique floue, ainsi que le déroulement et les usages sociaux de démos de dispositifs techniques, a permis de saisir dans un même mouvement l'implication et la coproduction d'un nouveau formalisme et de formes d'ordre technologique et social.

L'analyse des pratiques de la logique, celles d'écriture et de lecture bien sûr, mais surtout de démonstration, s'est révélée cruciale de ce point de vue. En effet, les exercices démonstratifs examinés engageaient, comme il a été vu, tout à la fois la construction de nouveaux objets formels, l'adhésion de lecteurs ou d'auditeurs à divers résultats, la reconnaissance de travaux, des chaînes de crédit, la formation d'espaces de collaboration et de concurrence et, plus ou moins directement, des formes de financement des activités de recherche, le développement et la vente de produits dits flous.

Certes, le terme «démonstration » a été mobilisé ici pour décrire des pratiques qui ne sont pas universellement célébrées comme telles, comme par exemple les démos. Mais cette extension sémantique ne constituait en aucune façon un "glissement » de sens qui nous aurait échappé, une reprise aveugle des catégories des acteurs, ou une quelconque facilité d'écriture. Il s'agissait au contraire de construire un outil pour appréhender

47. LÉvine, 1991. Pour l'étude d'autres cas de voyageurs au « regard perçant ", et cependant aveugles à ce qu'on leur montre, voir SCHAFFER, 1992, et AsHMORE, 1993.

48. Voir, également, MACKENZIE, 1993. Cet article décrit un cas où la mise en avant par des ingénieurs de propriétés formelles dans un dispositif informatique (un microprocesseur) a pu donner lieu à controverse. 
l'unité d'une démarche possédant plusieurs déclinaisons, la mise en jeu des diverses formes de démonstration dépendant de l'appréhension par les chercheurs de la pertinence de leur mobilisation singulière en fonction des situations et des interlocuteurs rencontrés (par exemple, démos pour intéresser des industriels dans certains cas de figure, productions scripturales dans des articles destinés aux pairs, etc.)

Cependant, dans la mesure où les pratiques démonstratives sont clairement apparues fonction des variations d'usages et des conditions matérielles évolutives de production d'abstractions, c'est une histoire sociale des formes de démonstration que ce type d'analyse met en perspective. En effet, si par exemple la démo constituait au début des années 1990 une forme de démonstration qui, à titre de substitution d'autres, semblait être de plus en plus mobilisée dans de nombreuses situations (colloques, par exemple, ou même encore soutenances de thèse en logique, aussi bien dans les pays anglo-saxons qu'en France), le suivi de l'évolution située de telles pratiques peut plus généralement donner matière à analyse sociohistorique.

L'objet n'est pas anodin. Dans le cas qui vient d'être étudié, l'exercice des démonstrations engageait pleinement les constructions d'objets des acteurs impliqués et déterminait en grande partie l'activité de ces derniers. Le développement d'une histoire sociale des formes de démonstration quant à elle n'engage sans doute rien de moins (et ce, tout particulièrement, dans le cas des sciences dites « déductives ${ }^{49}$ ») que la saisie des transformations de la production et des modes de travail scientifiques.

Claude Rosental (septembre 1998).

49. Pour ce qui est des sciences qualifiées d'« inductives ", voir Licoppe, 1996. 


\section{BIBLIOGRAPHIE}

ALKER (Hayward), 1982, «Standard logic versus dialectial logic. Which is better for scientific political discourse? ", communication au $12^{\mathrm{e}}$ congrès mondial de l'Association internationale de science politique, Rio de Janeiro, 8-15 août.

Archives du forum électronique d'Internet « comp.ai.fuzzy », articles $\mathrm{n}^{\mathrm{os}} 40,124,1555$, 2072. Coordonnées FTP : ftp.cs.cmu.edu; user/ai/pubs/news/comp.ai.fuzzy.

Ashmore (Malcolm), 1993, " The theatre of the blind. Starring a Promethean prankster, a phoney phenomenon, a prism, a pocket, and a piece of wood », Social Studies of science, vol. 23, p. 67-106.

BARWISE (Jon) et ETCHEMENDY (John), 1987, The Liar. An essay on truth and circularity, Oxford, Oxford University Press.

Black (Max), 1937, "Vagueness. An exercise in logical analysis », Philosophy of science, vol. 4, 4, oct., p. 427-455.

BLoor (David), 1981, "Hamilton and Peacock on the essence of algebra ", in Herbert MehrTens, Henk Bos et Ivo Schneider, éd., Social History of 19th century mathematics, Boston, Birkhäuser, p. 202-232.

Boureau (Alain), 1989, " Propositions pour une histoire restreinte des mentalités ", Annales. ESC, 6, nov.-déc., p. 1491-1504.

Boureau (A.), 1992, "L'adage Vox Populi, Vox Dei et l'invention de la nation anglaise (viI'-Xil' siècle) », Annales. ESC, 4-5, juil.-oct., p. 1071-1089.

BOUVERESSE (Jacques), 1976, Le Mythe de l'intériorité, expérience, signification et langage privé chez Wittgenstein, Paris, Minuit.

Brian (Éric), 1994, La Mesure de l'État. Administrateurs et géomètres au Xvirr siècle, Paris, Albin Michel.

BRIAN (É.), 1995, "Le livre des sciences est-il écrit dans la langue des historiens? ", in Bernard LEPETIT, éd., Les Formes de l'expérience. Une autre histoire sociale, Paris, Albin Michel, p. 85-98.

Claverie (Élisabeth), 1991, "Voir apparaître, regarder voir ", Raisons pratiques, vol. 2 , p. 1-19.

Coluins (Harry), 1985, Changing Order. Replication and induction in scientific practice, Londres, Sage.

Compte rendu daté du 15 mai 1991 de la réunion tenue le 19 avril 1991 à Paris, Club Crin Logique floue, Paris, Association Écrin.

DASTON (Lorraine), 1991, "Marvelous facts and miraculous evidence in early modern Europe ", Critical Inquiry, vol. 18, p. 93-124.

Dubors (Didier), Prade (Henri) et Smets (Philippe), 1994, «Partial truth is not uncertainty. Fuzzy logic versus possibilistic logic », IEEE Expert, août, p. 15-19.

DurкheIм (Émile), 1990, Les Formes élémentaires de la vie religieuse, Paris, Presses universitaires de France. 
ElKan (Charles), 1993, "The paradoxical success of fuzzy logic », Proceedings of AAAI 1993, Cambridge, MA, MIT Press, p. 698-703.

GAINEs (Brian), 1983, « Precise past, fuzzy future », International Journal of manmachines studies, 19 , p. 117-134.

Gaines (Brian) et Kohout (Ladislav), 1977, « The fuzzy decade. A bibliography of fuzzy systems and closely related topics ", International Journal of manmachine studies, 9, p. 1-68.

GoFFMAN (Erving), 1971, The Presentation of self in everyday life, Harmondsworth, Penguin.

GoLDSTEIN (Catherine), 1995, Un théorème de Fermat et ses lecteurs, Saint-Denis, Presses universitaires de Vincennes.

Goody (Jack), 1977, The Domestication of the savage mind, Cambridge, Cambridge University Press.

Hutchins (Edwin), 1995, Cognition in the wild, Cambridge, MA, MIT Press.

JACQ (François), 1996, Pratiques scientifiques, formes d'organisation et représentations politiques de la science dans la France de l'après-guerre. La « politique de la science " comme énoncé collectif (1944-1962), thèse de doctorat de sociologie, Paris, École nationale supérieure des mines de Paris.

Kosko (Bart), 1990, « Fuzziness versus probability », International Journal of general systems, vol. 17, p. 211-240.

Kosko (B.), 1992, Neural Networks and fuzzy systems, Englewood Cliffs, NJ, Prentice Hall.

Kosko (B.), 1993, Fuzzy Thinking. The new science of fuzzy logic, New York, Hyperion.

Kosko (Bart) et IsaKa (Satoru), 1993, «La logique floue », Pour la science, 191, sept., p. 62-68.

La Lettre du club logique floue, 1991, Paris, Association Écrin, 1, sept.

La Lettre des clubs Crin, 1992, Paris, Association Écrin, 8, oct.

KuHN (Thomas), 1983, La Structure des révolutions scientifiques, trad. Laure Mryer, Paris, Flammarion.

LaKatos (Imre), 1984, Preuves et réfutations. Essai sur la logique de la découverte mathématique, Paris, Hermann.

Largeault (Jean), 1993, La Logique, Paris, Presses universitaires de France.

LATOUR (Bruno), 1983, "Give me a laboratory and I will raise the world ", in Karin Knorr-Cetina et Michael Mulkay, éd., Science observed. Perspectives on the social studies of science, Londres, Sage, p. 141-170.

LÉvine (Jean), 1991, " Où sont passés les contrôleurs flous nippons? ", La Lettre de l'automatique, Fontainebleau, centre Automatique et Systèmes, École nationale supérieure des mines de Paris, 12, sept., p. 2-4.

LicopPE (Christian), 1996, La Formation de la pratique scientifique. Le discours de l'expérience en France et en Angleterre (1630-1820), Paris, La Découverte.

Livingston (Eric), 1985, The Ethnomethodological Foundations of mathematics, Londres, Routledge.

MAcKenzIE (Donald), 1993, « Negociating arithmetic, constructing proof. The sociology of mathematics and information technology ", Social Studies of science, vol. 23 , p. 37-65.

McNeill (Daniel) et Freiberger (Paul), 1992, Fuzzy Logic. The discovery of a revolutionary computer technology. And how it is changing our world, New York, Simon and Schuster.

Mialet (Hélène), 1994, Le Sujet de l'invention, thèse de doctorat de philosophie, Paris, université de Paris I Sorbonne. 
Pickering (Andrew) et Stephanides (Adam), 1992, « Constructing quaternions. On the analysis of conceptual practice ", in Andrew Pickering, éd., Science as practice and culture, Chicago, University of Chicago Press, p. 139-167.

Quine (Willard Van Orman), 1976, The Ways of paradox and other essays, Cambridge, MA, Harvard University Press.

Rosental (Claude), 1993, "An ethnography of the teaching of logic ", communication au colloque Historical Epistemology, Institute for the history of science, University of Toronto.

RoSENTAL (C.), 1996, L'Émergence d'un théorème logique. Une approche sociologique des pratiques contemporaines de démonstration, thèse de doctorat de sociologie, Paris, École nationale supérieure des mines de Paris.

Rotman (Brian), 1993, Ad Infinitum... The ghost in Turing's machine, Stanford, Stanford University Press.

SainsBury (Richard Mark), 1988, Paradoxes, Cambridge, Cambridge University Press.

Schaffer (Simon), 1992, "Self Evidence ", Critical Inquiry, vol. 18, Winter, p. 327-362.

Shapin (Steven) et Schaffer (Simon), 1985, Leviathan and the air-pump, Princeton, NJ, Princeton University Press.

SMETS (Philippe), 1991, "Varieties of ignorance and the need for well-founded theory ", Information Sciences, 57-58, p. 135-144.

Synthèse Technologies de l'information, 1989-1990, Paris, Aditech, vol. 1.

WARwick (Andrew), 1995, "The laboratory of theory or what's exact about the exact sciences? ", in M. Norton WISE, éd., The Values of precision-exactitude, Princeton, NJ, Princeton University Press, p. 311-351.

WITTGENSTEIN (Ludwig), 1976, Wittgenstein's Lectures on the foundations of the mathematics, éd. Cora Diamond, New York, Harvester.

ZADEH (Lotfi A.), 1965, « Fuzzy Sets ", Information \& control, 8, p. 338-353. 